\title{
EL SISTEMA DE BARRERAS TOBÁCEAS HOLOCENAS DE LAS PARRAS DE MARTÍN (CORDILLERA IBÉRICA, TERUEL)
}

\author{
M. RICO-HERRERO ${ }^{*}$, C. SANCHO-MARCÉN², M.C. ARENAS-ABAD², \\ M. VÁZQUEZ-URBEZ², B.L. VALERO-GARCÉS ${ }^{1}$ \\ ${ }^{1}$ Instituto Pirenaico de Ecología, CSIC, Campus Aula Dei, Av. Montañana 1005, 50059 Zaragoza. \\ ${ }^{2}$ Dpto. de Ciencias de la Tierra, Universidad de Zaragoza, Pedro Cerbuna 12, 50009 Zaragoza.
}

\begin{abstract}
RESUMEN. En el valle del río Las Parras (Cuenca alta del río Martín, Cordillera Ibérica turolense) aparecen importantes registros tobáceos holocenos. El perfil longitudinal del río muestra dos fuertes rupturas de pendiente coincidiendo con la salida de sendos estrechamientos conocidos como los hocinos de las Palomas y del Pajazo. En estos puntos se encuentran las acumulaciones tobáceas más potentes, 49 m en el Hocino de las Palomas y 26 m en el Hocino del Pajazo, con facies que indican un crecimiento rápido en un sistema de cascadas y barreras que se desarrollan vertical y lateralmente aguas abajo. El resto del tramo estudiado presenta pendientes suaves (2\%) con depósitos tobáceos fluvio-lacustres, de morfología aterrazada de gran desarrollo longitudinal y menor potencia (hasta $8 \mathrm{~m}$ en El Batán), asociados con represamientos. El conjunto morfosedimentario corresponde a un sistema fluvial escalonado compuesto por cascadas y áreas represadas. Los datos cronológicos indican que las tobas holocenas del río Las Parras se formaron entre 9.9 y $3.1 \mathrm{ka}$, con un periodo de mayor desarrollo entre 5.5 y $3.1 \mathrm{ka}$. En los últimos 2000 años se produce un descenso brusco en la actividad tobácea en la zona que coincide con un periodo de mínima insolación estival y probablemente con una menor disponibilidad hídrica en la zona.
\end{abstract}

\section{The Holocene tufa barrage system of Las Parras de Martín (Iberian Range, Teruel)}

ABSTRACT. A well-developed Holocene tufa system occurs in the headwaters of Las Parras River, Iberian Range (Teruel province). The longitudinal profile of the river shows two knickpoints downstream of two gorges known as Hocino de las Palomas and Hocino del Pajazo. The largest tufa build-ups in this area $149 \mathrm{~m}$ and $26 \mathrm{~m}$ thick) were found just downstream these two gorges and associated waterfalls. Barrage and cascade structures and facies suggest a rapid down-stream and vertical development of the tufa system. Between the knickpoints and upstream, the river valley displays a low-gradient (2\%) and tufa deposits occur as laterally extensive terraces, with variable thickness (up to $8.2 \mathrm{~m}$ in El Batán) and fluvio-lacustrine tufa sequences associated to dammed areas. The Las Parras 
Tufa system represents a depositional environment including stepped fluvial systems with barrage-cascade and associated dammed areas separated by low gradient fluvial stretches. According to the chronological data, the development of Las Parras Holocene tufa occurred between $9.9 \mathrm{ky}$ and $3.1 \mathrm{ky}$, with the highest growth rate from 5.5 to $3.1 \mathrm{ky}$, and a significant reduction of tufa activity during the last 2000 years. This evolution reflects the water availability in the area, and is coherent with changes in summer insolation during the Holocene.

Palabras clave: tobas, Holoceno, río Las Parras, Cordillera Ibérica.

Key words: tufa deposits, Holocene, Las Parras River, Iberian Range.

Enviado el 7 de noviembre de 2012 Aceptado el 15 de enero de 2013

* Correspondencia: Instituto Pirenaico de Ecología, CSIC, Campus Aula Dei, Av. Montañana 1005, 50059 Zaragoza. E-mail: mayterico@ipe.csic.es

\section{Introducción}

Los depósitos tobáceos (Ford y Pedley, 1996) representan la respuesta morfosedimentaria externa de la dinámica de los sistemas kársticos, altamente sensibles a las condiciones ambientales. Su mayor actividad viene regulada por la disponibilidad hídrica, el desarrollo de suelos y de la cobertera vegetal acompañante, que favorecen la infiltración de aguas químicamente activas. En este contexto la formación de tobas aparece asociada con descargas de agua saturadas en calcita. Durante el Cuaternario estas condiciones ambientales aparecen bien establecidas durante períodos interglaciares, de manera que las etapas de mayor actividad tobácea se correlacionan con Estadios Isotópicos Marinos impares, tanto a escala global (Henning et al., 1983) como regional (Durán, 1989). Los registros tobáceos constituyen, por tanto, excelentes indicadores paleoambientales y paleoclimáticos durante el Cuaternario.

El drenaje de la Cordillera Ibérica alberga importantes sistemas tobáceos cuaternarios de gran revelancia nacional e internacional. Sin duda, la presencia de extensas superficies aplanadas desarrolladas sobre calizas y compartimentadas por movimientos neotectónicos (Peña et al., 1984) hace que la red fluvial esté alimentada por descargas subterráneas kársticas y presente perfiles longitudinales con gradientes altos y rupturas marcadas. Así, en los cursos fluviales de orden menor, la desgasificación mecánica del agua favorece la nucleación y desarrollo de tobas con distintos dispositivos sedimentarios (p. ej. barreras y represamientos) (Arenas-Abad et al., 2010a; Vázquez-Urbez et al., 2012).

Concretamente, la red fluvial de la Cordillera Ibérica dirigida hacia el río Ebro presenta extensas y potentes construcciones tobáceas cuaternarias en los valles de los ríos Añamaza (Arenas et al., 2010b; Luzón et al., 2011; Vázquez-Urbez et al., 2011a, 2012), Mesa (Vázquez-Urbez, 2008), Piedra (Arenas et al., 2004; Sancho et al., 2010; Váz- 
quez-Urbez, 2008; Vázquez-Urbez et al., 2011 a y b, 2012) y Matarraña (MartínezTudela et al., 1986).

El valle del río Martín también presenta interesantes acumulaciones tobáceas cerca de Albalate del Arzobispo (Lozano et al., 2004) y Las Parras de Martín (Valero-Garcés et al., 2008a). Estos trabajos aportan los primeros datos cronológicos de estos sistemas fluviales tobáceos estructurados morfológicamente en niveles encajados. Se diferencian tres etapas de desarrollo tobáceo con edades de 175-145 ka, 44 ka y 7-4 ka, respectivamente. De todas ellas, los edificios correspondientes a la etapa holocena son los que presentan un mayor espesor y desarrollo espacial.

De cara a complementar las investigaciones anteriores, el objetivo de este trabajo consiste en valorar el contexto morfotopográfico, el ambiente sedimentario y el marco cronológico en el que se desarrollan las formaciones tobáceas holocenas aguas abajo de la población de Las Parras de Martín. Adicionalmente, se deduce información paleoambiental que sirve para mejorar el conocimiento del escenario paleoclimático regional durante el Holoceno.

\section{El área de estudio}

Las Parras de Martín es una pequeña localidad perteneciente al término municipal de Utrillas incluido en la comarca de Cuencas Mineras (provincia de Teruel). Se asienta al norte de la Sierra de San Just en la Cordillera Ibérica (Fig. 1A). El clima es mediterráneo seco-semiárido continentalizado con fuertes contrastes estacionales. La zona presenta una temperatura media anual de $12^{\circ} \mathrm{C}$ con medias invernales y estivales de $2^{\circ} \mathrm{C}$ y $18^{\circ} \mathrm{C}$ respectivamente. La precipitación total anual oscila entre 500 y $600 \mathrm{~mm}$. El relieve circundante (Fig. 1B) presenta fuertes contrastes topográficos (entre 1300 y $950 \mathrm{~m}$ ) relacionados con la estructura geológica y la incisión fluvial del río de Las Parras, pequeño afluente del río Martín.

El sustrato geológico (IGME, 1979) (Fig. 1C) está formado por materiales mesozoicos carbonatados del Jurásico inferior (dolomías y calizas), Cretácico inferior (calizas, margas, arenas y arcillas) y Cretácico superior (calizas y dolomías). Este conjunto se presenta intensamente deformado con estructuras tectónicas complejas de orientación NO-SE y NE-SO. Al norte y de manera discordante sobre las unidades anteriores aparecen conglomerados, calizas y lutitas oligocenas.

Desde el punto de vista geomorfológico, las zonas culminantes se encuentran arrasadas por la Superficie de Erosión Fundamental de la Cordillera Ibérica (Peña et al., 1984). La erosión diferencial durante el Cuaternario, asociada a la incisión de la red de drenaje, ha modelado una amplia variedad de relieves. Entre ellos destacan las barras estructurales, asociadas con capas verticales, que atraviesa el río de Las Parras, aguas abajo de la población de las Parras de Martín, conformando dos estrechos fluviales conocidos, localmente, como Hocino de las Palomas y Hocino del Pajazo. En este sector aparecen potentes depósitos de tobas asociados con el río de Las Parras, que constituyen el objeto de este estudio. 

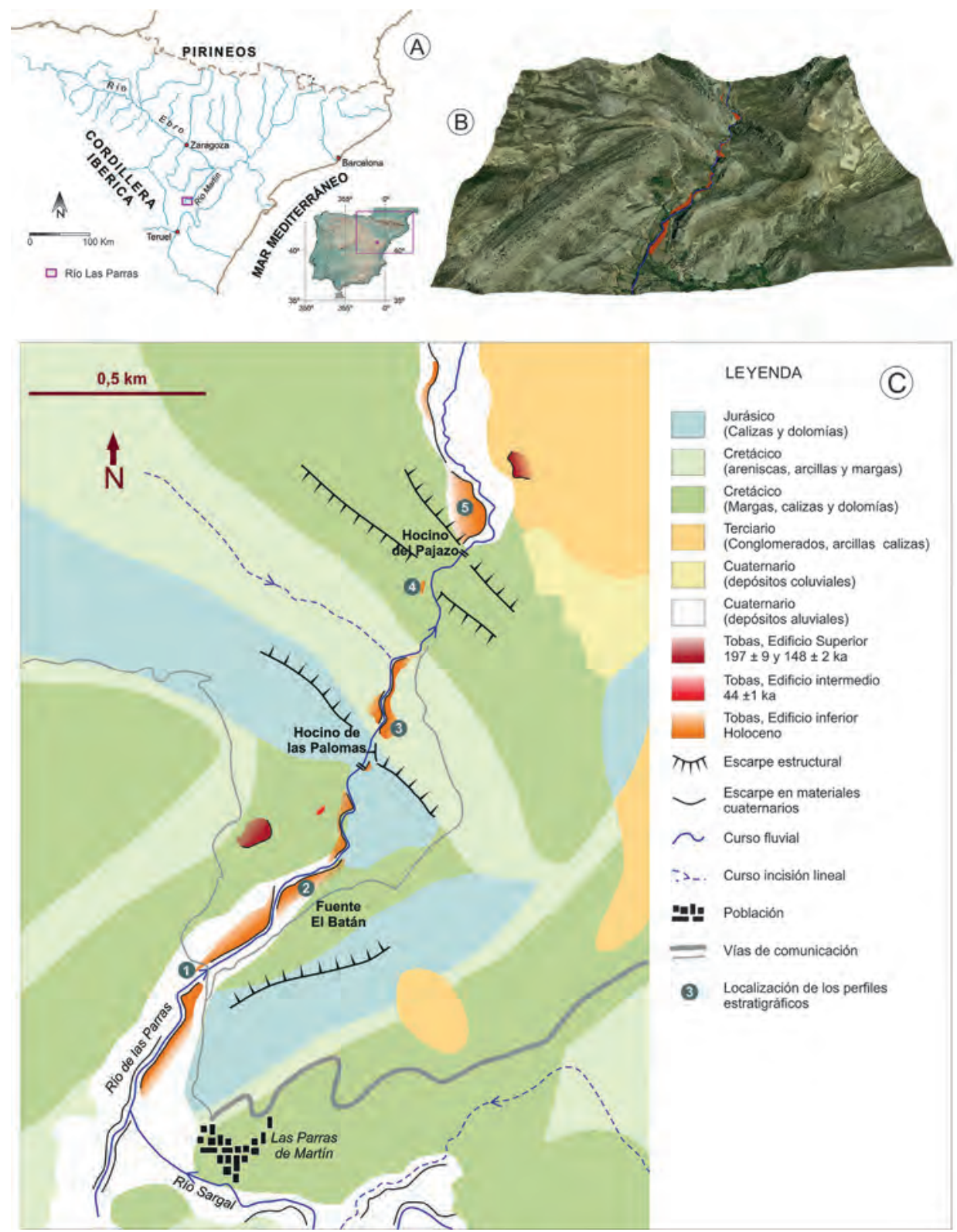

Figura 1. Mapa de situación del área de estudio (A), MDT del tramo de río en el que se localizan las tobas (B) y esquema geológico-geomorfológico y cartografía de las tobas holocenas $(C)$ con la localización de los perfiles estratigráficos: 1-Cruce sobre el río; 2- El Batán; 3- El Hocino de las Palomas (HPal en la margen izquierda y HPa2 en la margen derecha); 4- Puente entre los Hocinos; 5- El Hocino del Pajazo. 


\section{Metodología}

Aguas abajo de la población de Las Parras de Martín, se ha realizado una cartografía de los edificios tobáceos más recientes (Fig. 1C), a partir de fotografías aéreas, complementada con revisiones de campo. Paralelamente, se ha reproducido la geometría longitudinal de los edificios a partir de medidas altimétricas de precisión (datos obtenidos con un GPS Trimble GeoExplorer CE) de la superficie de los registros tobáceos y del perfil del río. Los datos tomados en campo se han corregido con una edición diferencial en postproceso para mejorar la precisión, utilizando como referencia los datos de la estación base de referencia del Gobierno de la Rioja. Tras realizar la corrección la precisión de los puntos oscila entre 0.4-1.2 m en la horizontal y 0.5-2 m en la vertical. Estos datos se han combinado con información derivada del modelo digital del terreno con paso de malla de 5 m (MDT5) del Centro Nacional de Información Geográfica.

El reconocimiento de campo ha permitido seleccionar afloramientos en los que levantar perfiles estratigráficos y muestrear restos de materia orgánica y trozos de carbón para realizar dataciones mediante radiocarbono. Las muestras se han tomado en niveles con alto contenido orgánico siguiendo criterios estratigráficos. En la terraza próxima a la fuente del Batán (Fig. 1C), en la margen derecha del río las Parras, se han seleccionado aquellos niveles de facies orgánicas más próximas a la base y techo de cada secuencia. En el caso de los edificios tobáceos de los hocinos del Pajazo y de las Palomas (Fig. 1C) se obtuvieron muestras procedentes de niveles próximos a la base y techo de la secuencia y muestras intermedias tomadas en niveles próximos a cambios importantes en el desarrollo de los edificios. Las dataciones se llevaron a cabo en el laboratorio de Beta Analytic Inc en Florida (USA) y el Poznań Radiocarbon Laboratory en Poznan (Polonia). La calibración de las edades se ha realizado mediante el programa CalPal, a través de su aplicación online (CalPal-2007 online; Danzeglocke et al., 2012) mediante la curva de calibración CalPal 2007 Hulu (Weninger y Jöris, 2008).

\section{Las tobas del río Las Parras}

\subsection{Distribución y geometría de los edificios tobáceos}

Las características del sustrato rocoso que atraviesa el río Las Parras han dado lugar a la formación de tramos encajados en los carbonatos, de alto gradiente, que han favorecido el desarrollo de importantes formaciones tobáceas durante el Cuaternario. Los edificios más importantes aparecen en relación con los estrechamientos del Hocino del Pajazo y del Hocino de las Palomas (Fig. 1C). Además, a lo largo del tramo comprendido entre la población de Las Parras de Martín y el Hocino de las Palomas, se encuentran edificios de morfología aterrazada en ambas márgenes del río, de gran desarrollo longitudinal pero de espesor reducido. La reconstrucción del perfil longitudinal de la superficie de la terraza tobácea reciente del río de Las Parras y su comparación con el perfil del río permite abordar la geometría longitudinal de los depósitos tobáceos y su relación con las discontinuidades morfotopográficas inducidas por la resistencia diferencial del sustrato geológico. 


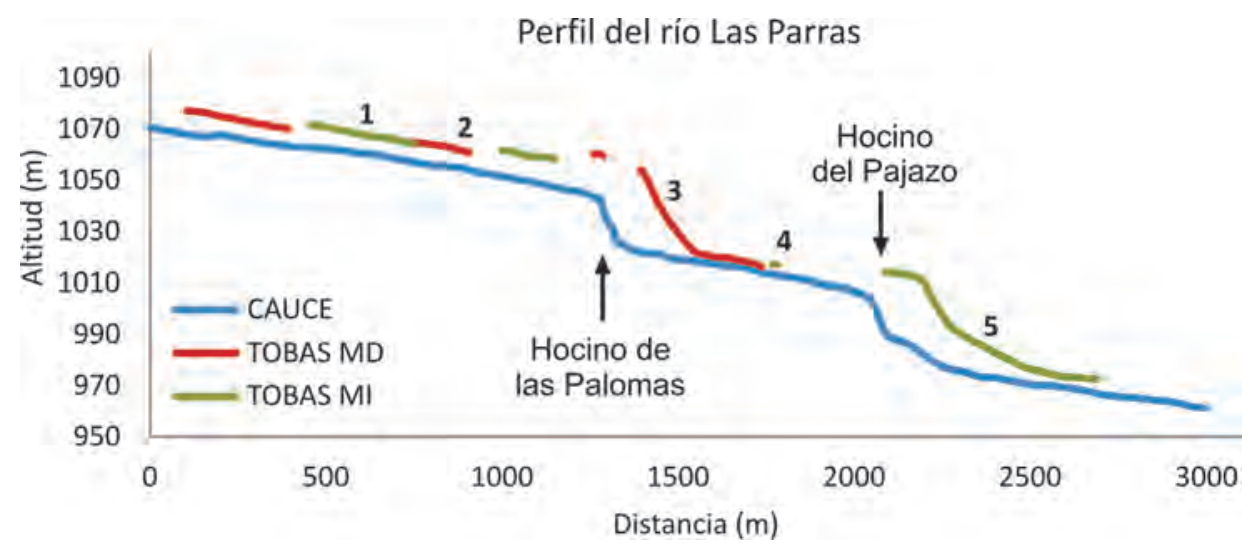

Figura 2. Corte longitudinal del perfil del río y de la superficie morfosedimentaria de la unidad de tobas holocenas. En verde se señalan las superficies localizadas en la margen izquierda (MI) y en rojo las situadas en la margen derecha $(M D)$. Se indica la localización de los perfiles estratigráficos (ver pie de Fig. 1).

El perfil longitudinal del río de las Parras presenta dos fuertes rupturas (Fig. 2). En el primer tramo el curso se adapta a la orientación de las estructuras tectónicas y presenta una pendiente baja (2.0\%). Cuando el río atraviesa transversalmente las dolomías y calizas del Jurásico inferior se conforma el Hocino de las Palomas y aparece la primera ruptura importante alcanzando una fuerte pendiente de hasta el 29\%. El afloramiento de las arenas y arcillas del Cretácico inferior permite al río recuperar su perfil de equilibrio (2.3\%). Sin embargo, cuando el río corta las capas verticales de calizas del Cretácico Superior modela el Hocino del Pajazo y aparece una nueva ruptura en el perfil longitudinal $(30.7 \%)$.

La superficie de la terraza tobácea se dispone paralela al perfil del río de Las Parras en los tramos interrupturas (Fig. 2). Las observaciones de campo indican que los depósitos tobáceos en dichos tramos presentan espesores reducidos (8.2 m en El Batán). Por otro lado, en los tramos de las rupturas, la superficie de la terraza aparece también paralela al perfil del río pero desplazada aguas abajo (Fig. 2). En estos sectores aparecen acumulaciones tobáceas, que alcanzan espesores mínimos de 49 m en el Hocino de las Palomas y de $26 \mathrm{~m}$ en el Hocino del Pajazo.

\subsection{Análisis estratigráfico}

Los cinco perfiles estratigráficos levantados en el área estudiada (Fig. 1C) registran un espesor variable entre 3 y $34 \mathrm{~m}$. Algunos se muestran de forma esquemática en la Fig. 3 , donde se sitúan las muestras recogidas para dataciones de radiocarbono.

Los perfiles de los hocinos del Pajazo (26 m) (Fig. 4) y de las Palomas 1 y 2 (15 y $34 \mathrm{~m}$ ) (Fig. 5) están formados por gravas y conglomerados en la base e incluyen clastos dispersos o alineados en algunos tramos (Fig. 4A). Los clastos son muy angulosos y 

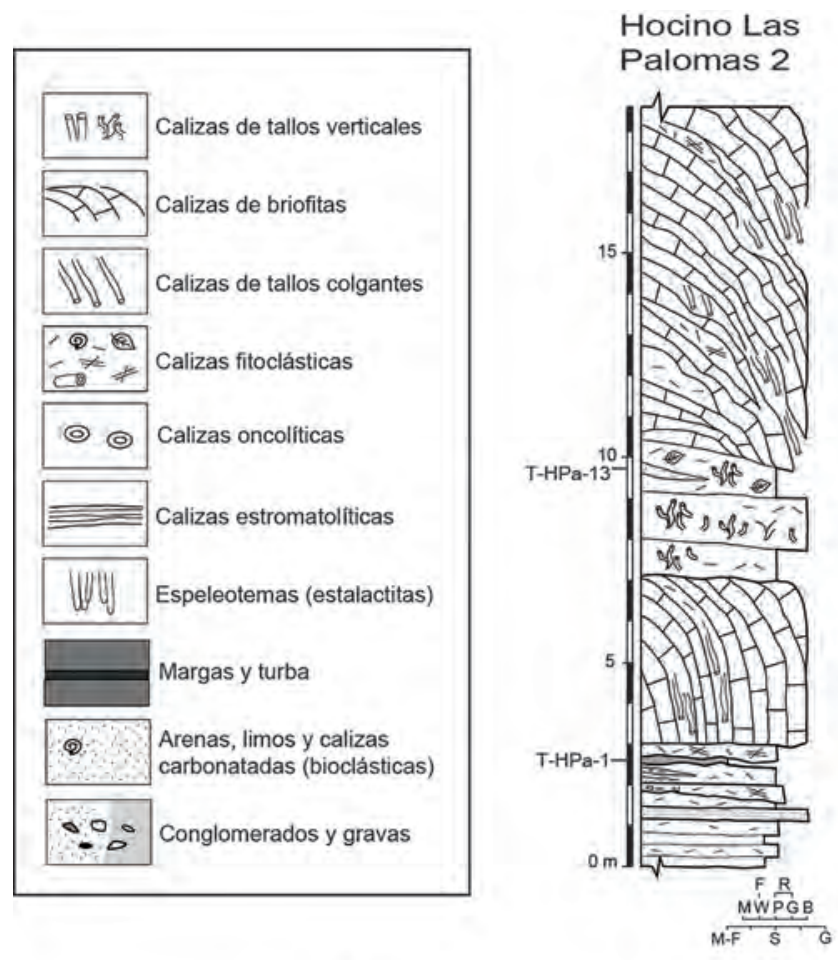

Hocino del Pajazo

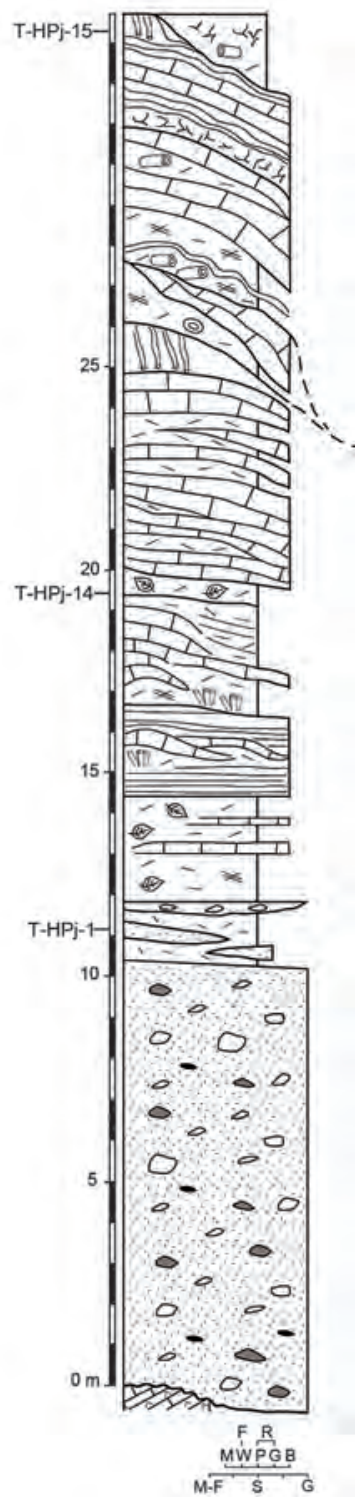

Figura 3. Perfiles estratigráficos esquemáticos de los depósitos Hocino de las Palomas 2, Hocino del Pajazo y El Batán, con la posición de muestras datadas. 

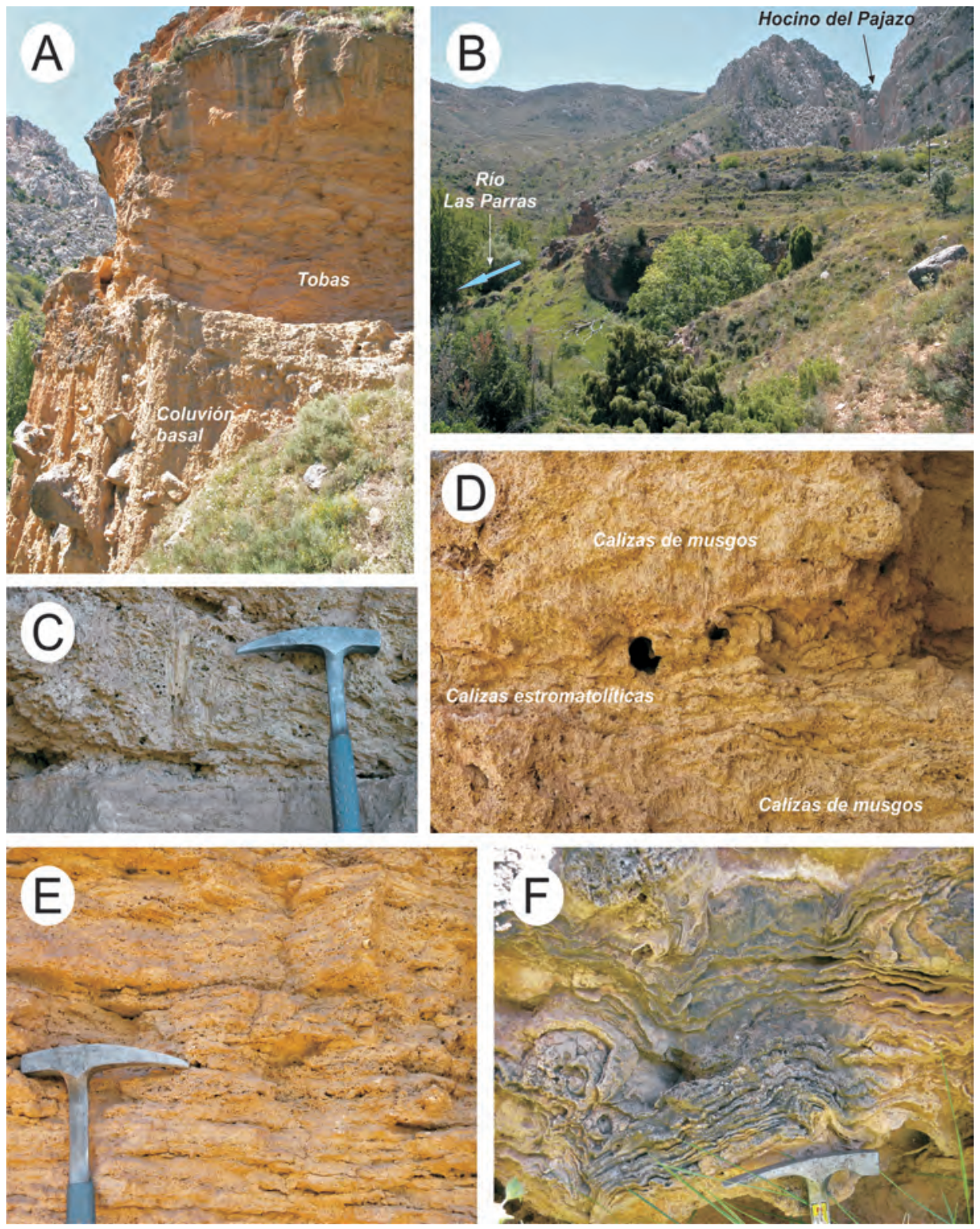

Figura 4. Depósitos del afloramiento El Pajazo. (A) Coluvión basal y depósitos bien estratificados de tobas. (B) Vista general de la parte superior del depósito El Pajazo en la margen izquierda del río Las Parras. Una flecha azul señala la dirección y sentido de la corriente. (C) Detalle de calizas fitoclásticas y calizas de tallos verticales (creciendo hacia arriba).(D) Detalle de la alternancia de intervalos de calizas de musgos y de calizas estromatolíticas.

(E) Calizas fitoclásticas finas laminadas y bandeadas con intercalaciones de calizas estromatolíticas. (F) Detalle de calizas estromatolíticas. 

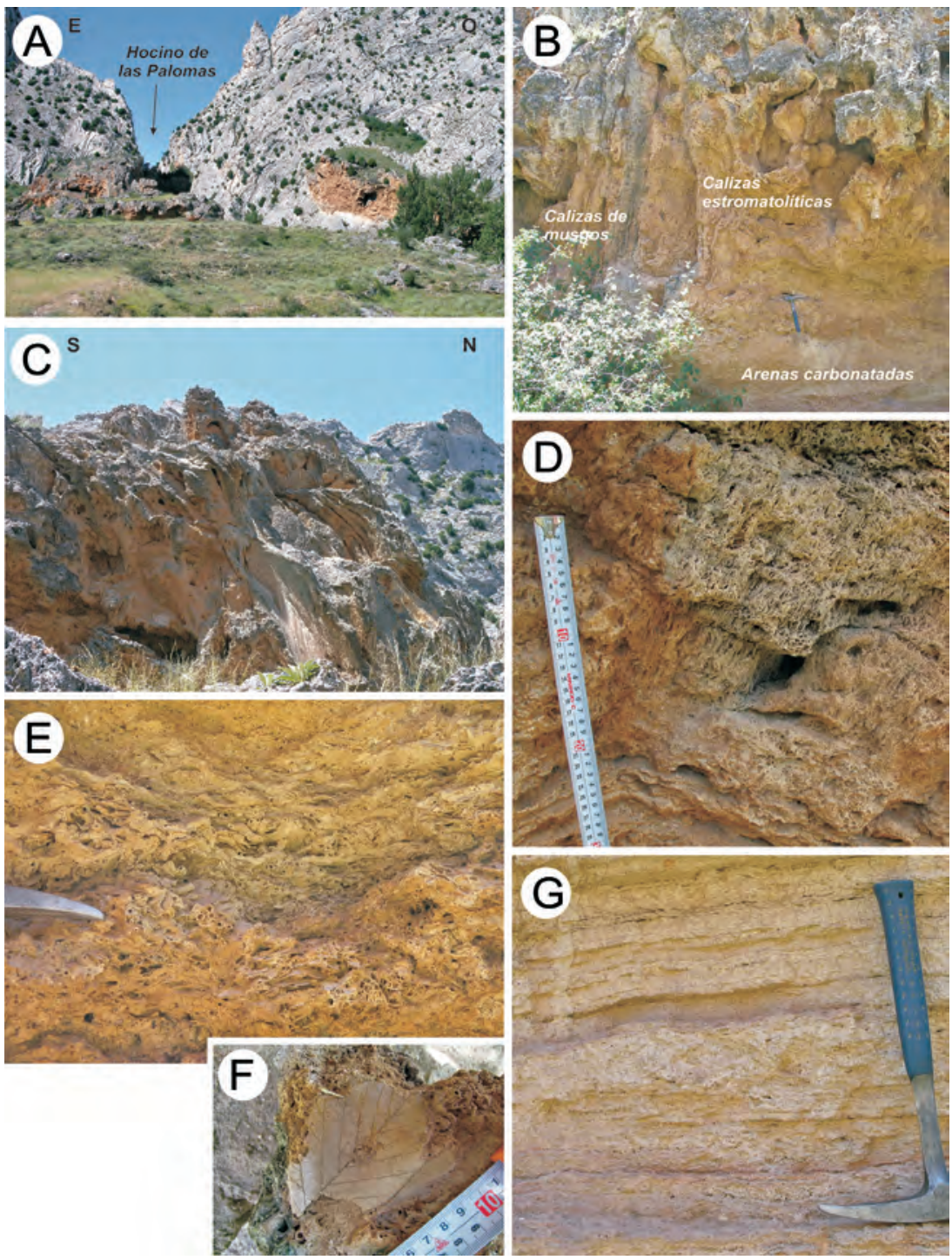

Figura 5. Depósitos del afloramiento Las Palomas. (A) Vista general hacia el sur que muestra la parte superior del edificio tobáceo separado en dos partes por el actual río Las Parras. (B) Depósitos de arenas carbonatadas bandeadas (parte inferior) sobre los que se disponen calizas de musgos (intervalos verticales a la izquierda) y calizas estromatolíticas y de tallos colgantes.

(C) Calizas de musgos, tallos colgantes y fitoclastos ( $p$. ej. zonas con cavidades circulares). Nótese la progradación hacia el norte. (D) Detalle de calizas de musgos mostrando sucesivas bandas onduladas en las que se aprecian los tallos de los musgos calcificados perpendiculares al bandeado. La parte inferior es una caliza fitoclástica. (E) Detalle de calizas fitoclásticas formadas por cortezas calcíticas alrededor de tallos y por abundantes moldes y hojas calcificadas. $(F)$ Detalle de una hoja. $(G)$ Calizas laminadas y bandeadas, formadas por facies fitoclásticas finas (más abundantes en la base y el centro) y estromatolíticas. 
heterométricos, derivados de las formaciones mesozoicas circundantes. El contenido en matriz (margas, arenas y limos) es variable, llegando a presentar fábrica no granosostenida. Por encima se encuentra una gran diversidad de calizas tobáceas, dispuestas en intervalos tabulares y lenticulares, algunos de los cuales configuran cuerpos hemidómicos de espesor métrico a decamétrico.

Entre las facies más abundantes se encuentran las calizas fitoclásticas (fragmentos de cortezas calcíticas alrededor de plantas, impresiones de plantas y hojas) (Figs. 4F y $5 \mathrm{E}, \mathrm{F})$, que a menudo alternan con calizas de musgos. El tamaño de estos fragmentos varía desde milimétrico a decimétrico (longitud). Los de menor tamaño suelen formar conjuntos laminados o bandeados (que incluyen intraclastos y peloides). Las calizas fitoclásticas pueden estar asociadas a superficies erosivas. También es frecuente encontrar calizas de tallos de plantas in situ (creciendo hacia arriba), constituyendo intervalos lenticulares de espesor centimétrico a $15 \mathrm{~cm}$; se conservan las cortezas calcíticas alrededor de los tallos, los cuales ya se han destruido; generalmente se disponen como arbustos o empalizadas (Fig. 4C).

Las calizas de musgos están dispuestas en láminas y bandas de varios centímetros (Figs. 4D y 5D), comúnmente con gran inclinación (5C), cuya acumulación puede constituir depósitos dómicos y hemidómicos de varios decímetros a decenas de metros de altura. A menudo, dentro de estos depósitos se encuentran intercalaciones de calizas de tallos colgantes (creciendo hacia abajo). Las calizas estromatolíticas aparecen en intervalos centimétricos a decimétricos, bien intercaladas en las calizas de musgos (Fig. 4D), bien alternando con calizas fitoclásticas (Figs. 4E y 5G). De manera ocasional, se reconocen lentículas centimétricas de margas grises y arenas carbonatadas cremas con gasterópodos, fitoclastos finos y fragmentos carbonosos (Fig. 5B).

En los perfiles El Batán, Puente entre los hocinos y Cruce sobre el río se han medido 8.2, 3 y $4 \mathrm{~m}$ de espesor, respectivamente. Se reconoce un nivel de gravas en la base del perfil El Batán (Fig. 6A), así como pequeñas intercalaciones lentículas de gravas y clastos dispersos en los otros dos perfiles. Las gravas tienen características similares a las de los perfiles de los hocinos descritas arriba. Los tres perfiles se caracterizan por el predominio de cuerpos tabulares horizontales, a veces con ligera inclinación, de hasta $1.2 \mathrm{~m}$ de espesor, formados por limos y arenas de composición carbonatada y margas, de color crema y gris (Figs. 6A, C). A menudo incluyen gasterópodos, bivalvos, fragmentos e impresiones de vegetales carbonosos y fitoclastos milimétricos o de pocos centímetros (Fig. 6C); más raramente incluyen escasos oncoides y nódulos de carbonato de escala milimétrica a centimétrica; en ocasiones los fitoclastos pueden ser el componente dominante. Son más raras las calizas de fitoclastos y las de tallos in situ (creciendo hacia arriba). Ocasionalmente aparecen lutitas carbonatadas ocres. Los depósitos de grano más fino se caracterizan por su color gris y marrón, a veces muy oscuro, y la inclusión de láminas y bandas centimétricas de color gris oscuro a negro ricas en materia orgánica (Fig. 6A, B). 

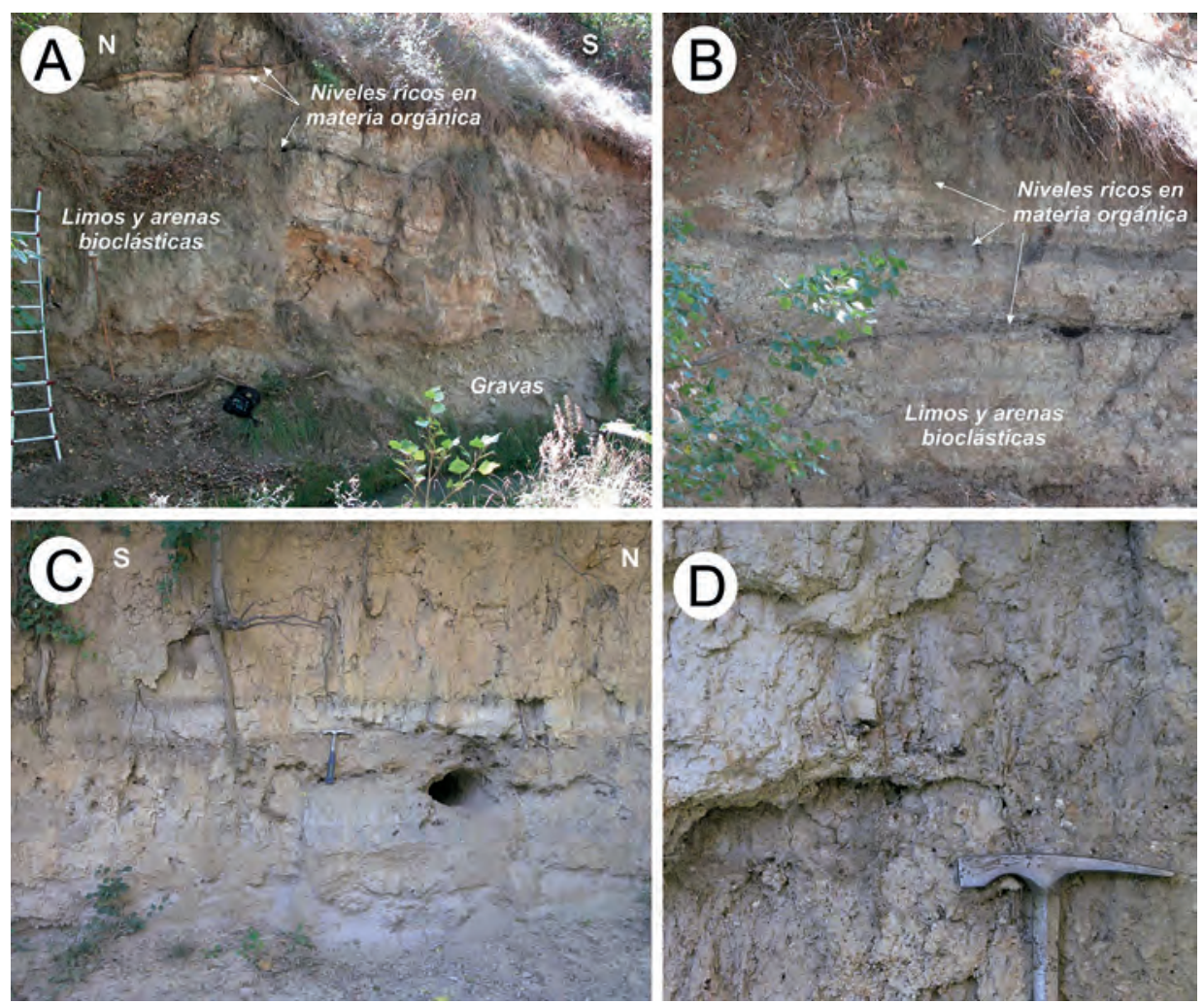

Figura 6. Depósitos de los afloramientos El Batán $(A, B)$ y Cruce sobre el río $(C, D)$. (A, B, C y $D)$ Depósitos tabulares de limos y arenas bioclásticas alternando con intervalos oscuros más ricos en materia orgánica carbonosa. (A) Corresponde a los $6 \mathrm{~m}$ inferiores del perfil y

(B) a los $3.3 \mathrm{~m}$ superiores del perfil El Batán (Fig. 3). Nótese en (A) el depósito de gravas en la base. (D) Detalle de (C).

Los afloramientos en los que se han levantado los perfiles de los hocinos del Pajazo y de las Palomas se interpretan, en conjunto, como depósitos formados en relación con tramos del río en donde la acumulación de fitoclastos puede generar pequeñas barreras y donde se desarrollan pequeños montículos de musgos. El crecimiento continuado de estos dispositivos estaría favorecido por la mayor velocidad del agua en las zonas de mayor pendiente (quizás en relación con rupturas de pendiente del sustrato, especialmente en las salidas del curso de agua de los estrechos) y, en definitiva, exageraría las irregularidades iniciales del sustrato. El proceso llevaría a la construcción de potentes depósitos de tobas en los que destacan las facies de barrera-cascada (calizas de musgos, tallos verticales y fitoclastos), especialmente en la parte superior.

Representan, por tanto, áreas con un incremento importante de la pendiente del río, en las que se formarían dispositivos de barrera-cascada, justamente en los tramos de estrechamiento y contacto entre formaciones geológicas de distinta resistencia. 
Por otro lado, el desarrollo de estromatolitos con intercalaciones de calizas fitoclásticas podría representar la sedimentación en tramos fluviales de pendiente suave, sin saltos importantes, pero con flujo rápido del agua. Las calizas fitoclásticas finas bandeadas también podrían originarse en zonas remansadas o represadas.

Por otra parte, los perfiles del Batán, Puente entre los hocinos y Cruce sobre el río representan, en conjunto, sedimentación en condiciones tranquilas, en agua con suave movimiento según la dirección de aportes o agua estancada. Esta situación favorecería el desarrollo de moluscos, así como de vegetación hidrófila y condiciones anóxicas. La mayor parte del sedimento procedería de la fragmentación de depósitos tobáceos formados aguas arriba, junto con la precipitación in situ. Durante los momentos de menor renovación del agua, las condiciones de anoxia favorecerían la conservación de materia orgánica procedente de plantas acuáticas (microscópicas y macroscópicas), produciendo importantes acumulaciones de turba. Las características de estos depósitos, junto con su localización entre los hocinos o aguas arriba del Hocino de las Palomas, permiten asignar su sedimentación a áreas represadas por los dispositivos de barrera-cascada anteriores.

\subsection{Datos cronológicos}

De los perfiles estratigráficos descritos se tomaron muestras para datar en tres de ellos: El Batán, el Hocino de las Palomas y el Hocino del Pajazo. Los resultados de las dataciones se muestran en la tabla 1.

Tabla 1. Datos de radiocarbono de los perfiles Hocino de las Palomas (T-HPa), Hocino del Pajazo (T-HPj) y El Batán (T-BAT). Las fechas han sido calibradas mediante la curva de calibración CalPal2007 Hulu (Weninger et al., 2007).

\begin{tabular}{|l|c|l|l|l|l|l|}
\hline Sigla & $\begin{array}{c}\text { Distancia respecto } \\
\text { a base perfil }\end{array}$ & Lab. $n o$. & Edad ${ }^{14}$ C & $\begin{array}{l}\text { Tipo de } \\
\text { muestra }\end{array}$ & $\begin{array}{l}{ }^{14} \text { C-Edad } \\
\text { cal BP }\end{array}$ & $\begin{array}{l}\text { Edad } \\
\text { calendario }\end{array}$ \\
\hline T-HPa1-03 & 1.1 & Poz-45343 & $8780 \pm 50$ & charcoal & $9803 \pm 97$ & $7853 \pm 97 \mathrm{BC}$ \\
\hline T-HPa1-09 & 10.8 & Poz-45345 & $4850 \pm 50$ & charcoal & $5574 \pm 66$ & $3624 \pm 66 \mathrm{BC}$ \\
\hline T-HPa2-1 & 2.6 & Poz-45346 & $3650 \pm 35$ & charcoal & $3990 \pm 66$ & $2040 \pm 66 \mathrm{BC}$ \\
\hline T-HPa2-13 & 27.8 & Poz-37953 & $3095 \pm 35$ & charcoal & $3318 \pm 42$ & $1368 \pm 42 \mathrm{BC}$ \\
\hline & & & & & & \\
\hline T-HPj-1 & 11.1 & Poz-37952 & $7480 \pm 50$ & charcoal & $8293 \pm 64$ & $6343 \pm 64 \mathrm{BC}$ \\
\hline T-HPj-14 & 19.2 & 301275 & $3900 \pm 40$ & charcoal & $4337 \pm 62$ & $2387 \pm 62 \mathrm{BC}$ \\
\hline T-HPj-15 & 33.2 & 301276 & $2990 \pm 30$ & charcoal & $3180 \pm 57$ & $1230 \pm 57 \mathrm{BC}$ \\
\hline & & & & & & \\
\hline T-BAT-1 & 1.0 & Poz-45349 & $7570 \pm 70$ & charcoal & $8374 \pm 59$ & $6424 \pm 59 \mathrm{BC}$ \\
\hline T-BAT-2 & 3.1 & Poz-45348 & $5390 \pm 40$ & charcoal & $6197 \pm 67$ & $4247 \pm 67 \mathrm{BC}$ \\
\hline T-BAT-5 & 7.1 & Poz-45347 & $4070 \pm 40$ & charcoal & $4606 \pm 114$ & $2656 \pm 114 \mathrm{BC}$ \\
\hline
\end{tabular}


En la terraza tobácea del paraje de la Fuente del Batán se dataron tres muestras (T-BAT-1, T-BAT-2 y T-BAT-5), localizadas a 1, 3.1 y 7.1 metros de la base, respectivamente (Fig. 3). Las dos muestras superiores se tomaron en niveles limosos y oscuros, con alto contenido en material orgánico, y que presentan una buena continuidad lateral en todo el afloramiento. La muestra T-BAT-1 se obtuvo de un nivel rico en materia orgánica, poco continuo, localizado en el contacto entre las gravas basales y las tobas. De acuerdo con la datación obtenida para esta muestra, el comienzo de la formación de tobas en este tramo del río las Parras estaría fechado en $8374 \pm 59$ cal BP. Por otro lado, el nivel orgánico más alto que se ha datado (BAT-5), a unos $1.1 \mathrm{~m}$ del techo de la terraza, fecha el final de la secuencia posterior hace aproximadamente 4600 años.

En el perfil de las Palomas, se tomaron 4 muestras para su datación radiométrica (ver Tabla 1): una en la base del depósito de tobas $(1.1 \mathrm{~m})$ (T-HPa-03), una segunda situada a unos 10.8 metros de la base del perfil (T-HPa-09), ambas tomadas en el tramo inferior del edificio tobáceo y otras dos muestras más, T-HPa-13 y T-HPa-1, del tramo superior, situadas a 2.6 y $27.8 \mathrm{~m}$ de la base del perfil HPa2 (Fig. 3). Los resultados obtenidos indican que la formación de este potente depósito tobáceo comenzó en 9803 × 97 y continuó de forma discontinua durante un mínimo de 6500 años.

En el edificio asociado al Hocino del Pajazo se han datado en total tres muestras de carbón. Las muestras correspondientes a la base y techo del depósito de tobas (T-HPj-1 y T-HPj-15) indican una edad para las tobas de entre $8293 \pm 64$ y $3180 \pm 57{ }^{14} \mathrm{C}$ cal BP. La datación de la muestra intermedia indica edades de $4508 \pm 55$ y $4337 \pm 62{ }^{14} \mathrm{C}$ cal $\mathrm{BP}$, respectivamente. Lo que sugiere la existencia de un periodo con mayor desarrollo de las tobas entre hace aproximadamente 5000 y 3000 años.

\section{Discusión: nucleación y desarrollo de las barreras}

Los edificios holocenos del río de Las Parras se nuclean en los puntos de ruptura del perfil longitudinal del río que coinciden con discontinuidades litoestructurales introducidas por el sustrato geológico. En estos puntos se produce un incremento súbito de la turbulencia y agitación del agua, que conducen a su desgasificación mecánica (pérdida de $\mathrm{CO}_{2}$ disuelto), favoreciendo la precipitación del carbonato. En general, existe una estrecha correspondencia entre discontinuidades morfotopográficas y formación de tobas en los sistemas tobáceos fluviales (Meléndez et al., 1996; Sancho et al., 1997; Peña et al., 2000; Arenas et al., 2004, 2010a; Lozano et al., 2012; Vázquez-Urbez et al., 2011a,b, 2012).

Estos edificios tobáceos crecen muy rápidamente, dando lugar a sistemas de cascadas y barreras que se desarrollan vertical y lateralmente aguas abajo. Este acrecimiento vertical conlleva la aparición de áreas represadas asociadas aguas arriba de las barreras (Pedley, 1990; Pedley et al., 2003; Arenas-Abad et al., 2010a). En el caso del río de las Parras, se desarrollaron dos importantes edificios de barrera-cascada coincidiendo con la salida de los hocinos de las Palomas y del Pajazo. Estos edificios se 
caracterizan por presentar cuerpos tabulares ligeramente inclinados y cuñas, que en conjunto representan dispositivos de progradación y agradación. Aguas abajo de estos dispositivos podrían existir zonas encharcadas, así como tramos de río con escasa pendiente.

Por otro lado, el desarrollo de la barrera del Hocino de las Palomas generó un área de aguas represadas y encharcamientos que ocuparon el fondo del valle fluvial hasta Las Parras de Martín. En el tramo comprendido entre los dos hocinos también aparecen restos de represamientos asociados, en esta ocasión, con el Hocino del Pajazo. En estos ambientes, con aguas estancadas o moviéndose a baja velocidad, aparecerían moluscos y otros animales acuáticos, así como áreas ocupadas por la vegetación hidrófila. En las etapas de escasa renovación del agua se alcanzarían condiciones pantanosas, que propiciarían la formación de acumulaciones de turba.

Se podría pensar, en definitiva, en un sistema fluvial escalonado de lagos poco profundos separados por cascadas de agua. Las Lagunas de Ruidera (Ordóñez et al., 1986; Pedley et al., 1996) y los Lagos de Plitvice (Emeis et al., 1987) pueden ser considerados escenarios comparables de funcionalidad actual, si bien de dimensiones diferentes.

De acuerdo con los datos cronológicos obtenidos, el sistema tobáceo holoceno del río de Las Parras se desarrolla entre $3.1 \mathrm{ka} \mathrm{y} 9.9 \mathrm{ka}$. Durante el periodo comprendido entre aproximadamente 5.5 y $3.1 \mathrm{ka}$ se produce al mayor desarrollo de los edificios de barrera-cascada, representados en los hocinos del Pajazo y de las Palomas. Sería necesario datar nuevas muestras en los diferentes depósitos para constatar la posible presencia de diversos pulsos de crecimiento en este sistema.

Desde un punto de vista regional, durante el Holoceno se registra una fase de elevada actividad tobácea a escala mediterránea en los diferentes sistemas fluviales que drenan la Cordillera Ibérica hacia las cuencas de los ríos Ebro (Martínez-Tudela et al., 1986; Arenas et al., 2004, 2010a,b; Valero-Garcés et al., 2008a; Sancho et al., 2010; Vázquez-Urbez et al., 2011a), Tajo (Ordoñez et al., 1987, 1990; Torres et al., 2005; Valero-Garcés et al., 2008b; Ortiz et al., 2009; Domínguez-Villar et al., 2011) y cuencas mediterráneas (Sancho et al., 1997; Peña et al., 2000; Lozano et al., 2012).

Por analogía con interpretaciones derivadas del estudio de otros registros tobáceos (Henning et al., 1983; Vaudour, 1986; Goudie et al., 1993; García del Cura et al., 1997; Martín-Algarra et al., 2003; Ordoñez et al., 2005; Domínguez-Villar et al., 2011), las tobas holocenas asociadas con el río de Las Parras indican que entre 3 ka y $10 \mathrm{ka}$, en este sector de la Ibérica, el escenario paleoambiental estaba conformado por un paisaje biostático estabilizado caracterizado por una cobertera vegetal arbórea consolidada, suelos bien desarrollados y altas descargas de agua subterránea químicamente activa. Estas circunstancias requieren de condiciones húmedas y cálidas, superiores a las actuales. La ausencia de registro tobáceo durante los últimos 2-3 ka, indicaría un descenso en la acti- 


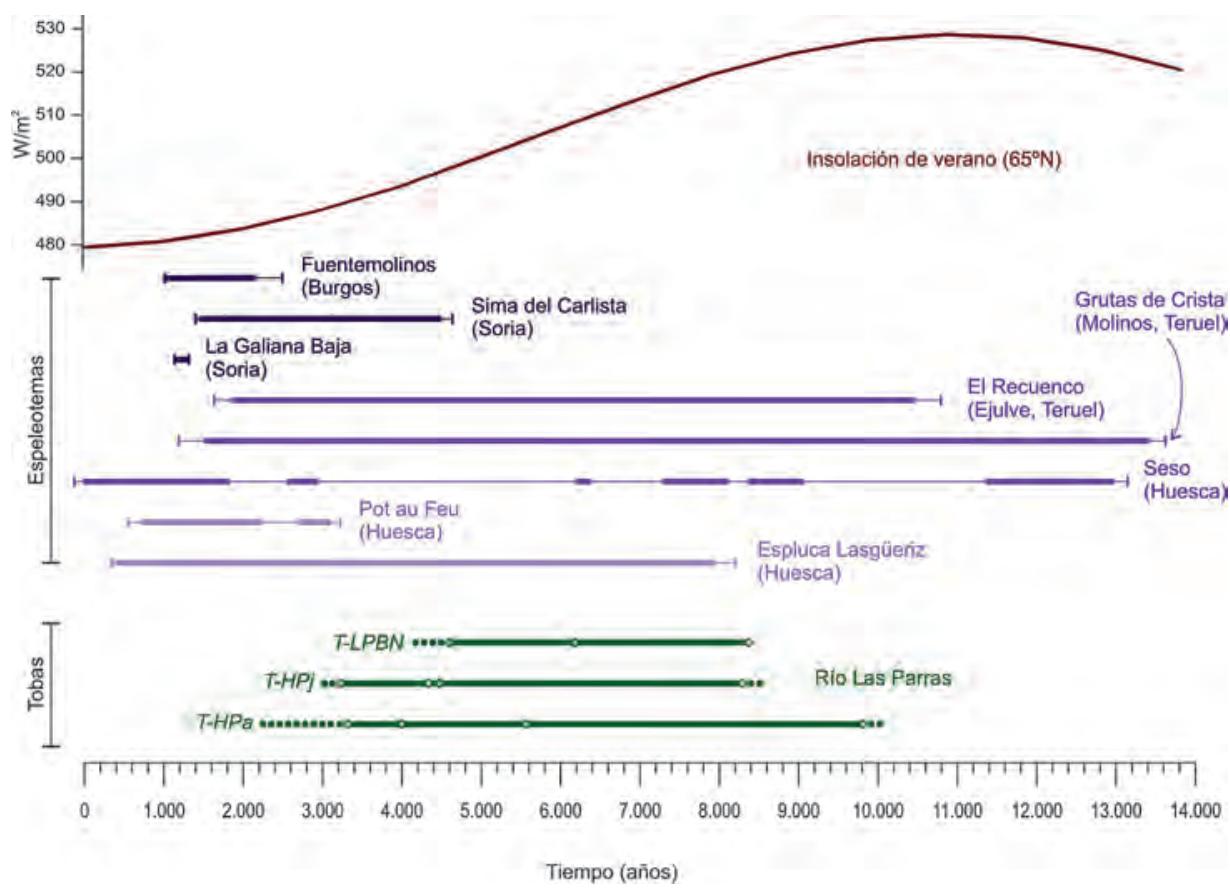

Figura 7. Fases de crecimiento de tobas en el río las Parras durante el Holoceno. En la parte superior se representa la curva de insolación de verano $\left(65^{\circ} \mathrm{N}\right)$ (Berger, 1978). En la parte central se han representado las fases de crecimiento de espeleotemas holocenos en el noreste peninsular (Moreno et al., este volumen).

vidad tobácea en respuesta a una menor disponibilidad hídrica coincidiendo con un mínimo en la insolación de verano (Fig. 7).

Este escenario es acorde con el mostrado a partir del estudio de la formación de espeleotemas en el Noreste Peninsular realizado por Moreno et al. (este volumen). Si bien se observa un desarrollo generalizado y bastante continuo de espeleotemas durante buena parte del Holoceno, en el Sistema Ibérico no se registra crecimiento espeleotémico durante el tramo final del Holoceno, lo cual ha sido interpretado por los autores como un descenso en la disponibilidad hídrica, que afectaría especialmente a las precipitaciones de influencia Mediterránea.

Aunque algunos sistemas fluviales de la Cordillera Ibérica presentan una elevada tasa de funcionalidad tobácea actual (Arenas et al., 2004, 2010c; Vázquez-Urbez et al., 2010, 2011a), en general los edificios tobáceos holocenos han sido incididos de manera generalizada por la red de drenaje, indicando un cambio en las condiciones climáticas y/o una influencia antrópica (Goudie et al., 1993) durante el Holoceno superior. El techo de la terraza tobácea holocena del río de Las Parras indica que la sedimentación tobácea cesó aproximadamente hace 2000 años, dando paso a la incisión fluvial y degradación de los edificios previamente construidos. 


\section{Agradecimientos}

Este trabajo ha sido financiado por los proyectos CGL2009-10455/BTE, CGL200909216/CLI, el proyecto "Clima y patrones de ocupación humana en las sierras turolenses durante el holoceno temprano" (PI089/09) financiado por el Gobierno de Aragón y por los Grupos de Investigación Paleoambientes del Cuaternario y Análisis de Cuencas Sedimentarias Continentales. Los autores agradecen a Miguel Sevilla su soporte técnico en el análisis de los datos topográficos.

\section{Referencias bibliográficas}

Arenas, C., Auqué, L.F., Elías, M.J., Longares, L.A., Osácar, M.C., Pardo, G., Peña, J.L., Sancho, C., Vázquez-Urbez, M. 2004. Depósitos Tobáceos actuales y fósiles del río Piedra (provincia de Zaragoza). En Geo-Guías 1. Itinerarios Geológicos por Aragón, F. Colombo, C.L. Liesa, G. Meléndez, A. Pocoví, C. Sancho, A.R. Soria (eds.), Sociedad Geológica de España, Zaragoza, pp. 11-32.

Arenas-Abad, C., Vázquez-Urbez, C., Pardo-Tirapu, G., Sancho-Marcén, C. 2010a. Fluvial and associated carbonate deposits. En Carbonates in continental settings. Facies, environments and processes, A.M. Alonso-Zarza, L.H. Tanner (eds.), Developments in Sedimentology, 61, Elsevier, Amsterdam, pp. 133-176.

Arenas, C., Sancho, C., Vázquez-Urbez, M., Pardo, G., Hellstrom, J., Ortiz, J.E., Torres, T., Osácar, C., Auqué, L. 2010b. Las tobas cuaternarias del río Añamaza (provincia de Soria, Cordillera Ibérica): aproximación cronológica. Geogaceta 49, 51-54.

Arenas, C., Osácar, C., Sancho, C., Vázquez-Urbez, M., Auqué, L., Pardo, G. 2010c. Seasonal record of recent fluvial tufa deposits (Monasterio de Piedra, NE Spain): sedimentation rates and stable isotope data. En Tufas and Speleothems: Unravelling the Microbial and Physical Controls, H.M. Pedley, M. Rogerson (eds.), Special Publication of the Geological Society, London, pp. 119-142.

Berger, A. 1978. Long-term variations of caloric insolation resulting from the Earth's orbital elements. Quaternary Research 9, 139-167.

Danzeglocke, U., Jöris, O., Weninger, B. 2012. CalPal-2007online. Disponible en: http://www. calpal-online.de/ (fecha de acceso: 13 de noviembre de 2012).

Domínguez-Villar, D., Vázquez-Navarro, J.A., Cheng, H., Edwards, R.L. 2011. Freshwater tufa record from Spain supports evidence for the past interglacial being wetter than the Holocene in the Mediterranean region. Global and Planetary Change 77, 129-141.

Durán, J.J. 1989. Riesgos geológicos ligados al karst en España. En El karst en España, J.J. Durán, J. Martínez (eds.), Sociedad Española de Geomorfología, Madrid, pp. 243-256.

Emeis, K.C., Richnow, H.H., Kempe, S. 1987. Travertine formation in Plitvice National Park, Yugoslavia: chemical versus biological control. Sedimentology 34, 595-609.

Ford, T.D., Pedley, H.M. 1996. A review of tufa and travertine deposits of the world. Earth-Science Reviews 41, 117-175.

García del Cura, M.A., González Martín, J.A., Ordóñez, S., Pedley, H.M. 1997. Las Lagunas de Ruidera. En Elementos del Medio Natural en la Provincia de Ciudad Real, J.L. García Rayego, E. González Cárdenas (eds.), Colección Estudios, Universidad de Castilla-La Mancha, Cuenca, pp. 83-129.

Goudie, A.S., Viles, H.A., Pentecost, A. 1993. The late-Holocene tufa decline in Europe. Holocene 3, 181-186. 
Henning, G.J., Grun, R., Brunnacker, K. 1983. Speleothems, travertins and paleoclimates. Quaternary Research 20, 1-29.

IGME 1979. Mapa Geológico de España. Hoja 517 Argente. IGME, Madrid.

Lozano, M.V., Peña, J.L., Longares, L.A., Sánchez, M. 2004. Cañones del río Martín entre Oliete y Albalate del Arzobispo (Cordillera Ibérica, Teruel). En Geografía física de Aragón. Aspectos generales y temáticos, J.L. Peña, L.A. Longares, M. Sánchez, M. (eds.), Universidad de Zaragoza e Institución Fernando el Católico, Zaragoza, pp. 213-230.

Lozano, M.V., Sancho, C., Arenas, C., Vázquez-Urbez, M., Ortiz, J.E., De Torres, T., Pardo, G., Osácar, M.C., Auqué, L. 2012. Análisis preliminar de las tobas cuaternarias del río Ebrón (Castielfabib, Valencia, Cordillera Ibérica). Geogaceta 51, 51-54.

Luzón, M.A., Pérez, A., Borrego, A.G., Mayayo, M.J., Soria, A.R. 2011. Interrelated continental sedimentary environments in the central Iberian Range (Spain): Facies characterization and main palaeoenvironmental changes during the Holocene. Sedimentary Geology 239, 87-103.

Martín-Algarra, A., Martín-Martín, M., Andro, B., Julià, R., González-Gómez, C. 2003. Sedimentary patterns in perched spring travertines near Granada (Spain) as indicators of the palehydrological and paleoclimatological evolution of a karst massif. Sedimentary Geology 161, 217-228.

Martínez-Tudela, A., Cuenca, F., Santisteban, C., Grun, R., Hentzsch, B. 1986. Los travertinos del Río Matarraña, Beceite (Teruel) como indicadores paleoclimáticos del Cuaternario. En Quaternary Climate in Western Mediterranean, A. López-Vera (ed.), Universidad Autónoma de Madrid, Madrid, pp. 307-324.

Meléndez, A., Peña, J.L., Sancho, C. 1996. Factores fisicoquímicos que intervienen en el desarrollo de las barreras tobáceas bioconstruidas del río de la Fuente del Berro (Sierra de Albarracín, Teruel). Revista de la Sociedad Geológica de España 9, 125-134.

Moreno, A., Belmonte, A., Bartolomé, M., Sancho, C., Oliva, B., Stoll, H., Edwards, L.R., Cheng, H., Hellstrom, J. 2013. Formación de espeleotemas en el Noreste Peninsular y su relación con las condiciones climáticas durante los últimos ciclos glaciares. Cuadernos de Investigación Geográfica 39 (1), 25-47.

Ordóñez, S., González, J.A., García del Cura, M.A. 1986. Petrography and morphology of Quaternary tufa structures in central Spain. Mediterranée 57, 52-60.

Ordóñez, S., González, J.A., García del Cura, M.A. 1987. Formaciones travertínicas y tobáceas en el valle del Tajo (Sector Cifuentes-Trillo): Estudio geomorfológico, petrológico y sedimentológico. Cuaternario y Geomorfología 1, 231-245.

Ordóñez, S., González, J.A., García del Cura, M.A. 1990. Datación radiogénica (U-234/U-238 y Th-230/U-234) de sistemas travertínicos del Alto Tajo (Guadalajara). Geogaceta 8, 53-56.

Ordóñez, S., González-Martín, J.A., García del Cura, M.A., Pedley, M. 2005. Temperate and semi-arid tufas in the Pleistocene to recent fluvial barrage system in the Mediterranean area: the Ruidera Lakes Natural Park (central Spain). Geomorphology 69, 332-350.

Ortiz, J.E., Torres, T., Delgado, A., Reyes, E., Díaz-Bautista, A. 2009. A review of the Tagus river tufa deposits (Central Spain): age and palaeoenvironmental record. Quaternary Science Reviews 28, 947-963.

Pedley, H.M. 1990. Classification and environmental models of cool freshwater tufas. Sedimentary Geology 68, 143-154.

Pedley, H.M., Andrews, J., Ordóñez, S., García del Cura, M.A., González-Martín, J.A., Taylor, D. 1996. Does climate control the morphological fabric of freshwater carbonates? A comparative study of Holocene barrage tufas from Spain and Britain. Palaeogeography, Palaeoclimatology, Palaeoecology 121, 239-257. 
Pedley, H.M., González-Martín, J.A., Ordóñez, S., García del Cura, M.A. 2003. Sedimentology of Quaternary perched spring line and paludal tufas: criteria for recognition, with examples from Guadalajara Province, Spain. Sedimentology 50, 23-44.

Peña, J.L., Gutiérrez, M., Ibáñez, M.J., Lozano, M.V., Rodríguez, J., Sánchez, M., Simón, J.L., Soriano, A., Yetano, L.M. 1984. Geomorfología de la provincia de Teruel. Instituto de Estudios Turolenses, Teruel, $149 \mathrm{pp}$.

Peña, J.L., Sancho, C., Lozano, M.V. 2000. Climatic and tectonic significance of Pleistocene and Holocene tufa deposits in the Mijares River canyon, eastern Iberian range, Northeastern Spain. Earth Surface Processes and Landforms 25, 1403-1417.

Sancho, C., Peña, J.L., Meléndez, A. 1997. Controls on Holocene and present-day travertine formation in the Guadalaviar River (Iberian Chain, NE Spain). Zeitschrift fur Geomorphologie, 41, 289-307.

Sancho, C., Arenas, C., Pardo, G., Vázquez, M., Hellstrom J., Ortiz, J.E., Rhodes, E.J., Osácar, C., Auqué, L. 2010. Ensayo cronológico de las tobas cuaternarias del río Piedra (Cordillera Ibérica). Geogaceta 48, 31-34.

Torres, T., Ortiz, J.E., García de la Morena, M.A., Llamas, F.J., Goodfriend, G. 2005. Ostracodebased aminostratigraphy and aminochronology of tufa system in central Spain. Quaternary International 135, 21-33.

Valero-Garcés, B.L., Moreno, A., Morellón, M., Corella, J.P., González-Sampériz, P., Mata, P. 2008a. Cronología de las tobas de ladera del río de Las Parras (Cordillera Ibérica, Teruel). En Trabajos de Geomorfología en España, 2006-2008, J. Benavente, J. Gracia, (eds.), Sociedad Española de Geomorfología, Madrid, pp. 71-74.

Valero-Garcés, B.L., Moreno, A., Navas, A., Mata, P., Machín, J., Delgado-Huertas, A., GonzálezSampériz, P., Schwalb, A., Morellón, M., Cheng, H., Edwards, R.L. 2008b. The Taravilla lake and tufa deposits (Central Iberian Range, Spain) as palaeohydrological and palaeoclimatic indicators. Palaeogeography, Palaeoclimatology, Palaeoecology 259, 136-156.

Vaudour, J. 1986. Travertins holocènes et pression anthropique. Méditerranée 1-2, 168-173.

Vázquez-Urbez, M. 2008. Caracterización y significado ambiental de depósitos tobáceos neógenos de la Cuenca del Ebro. Comparación con ambientes Cuaternarios. Tesis doctoral. Universidad de Zaragoza, Zaragoza, $476 \mathrm{pp}$.

Vazquez-Urbez, M., Arenas, C., Sancho, C., Osácar, C., Auqué, L. Pardo, G. 2010. Factors controlling present-day tufa dynamics in the Monasterio de Piedra Natural Park (Iberian Range, Spain): depositional environmental settings, sedimentation rates and hydrochemistry. International Journal of Earth Sciences (Geologische Rundschau) 99, 1027-1049.

Vázquez-Urbez, M., Arenas, C., Sancho, C., Auqué, L., Osácar, C., Pardo, G. 2011a. Quaternary and present-day tufa systems of the Rivers Piedra and Añamaza (Iberian Range, Spain). Geo-Guías 8, 241-274.

Vázquez-Urbez, M., Pardo, G., Arenas, C., Sancho, C. 2011b. Fluvial diffluence episodes reflected in the Pleistocene tufa deposits of the River Piedra (Iberian Range, NE Spain). Geomorphology 125, 1-10.

Vázquez-Urbez, M., Arenas, C., Pardo, G. 2012. A sedimentary facies model for stepped, fluvial tufa systems in the Iberian Range (Spain): the Quaternary Piedra and Mesa valleys. Sedimentology 59, 502-526

Weninger, B., Jöris, O. 2008. A ${ }^{14} \mathrm{C}$ age calibration curve for the last $60 \mathrm{ka}$ : the Greenland-Hulu $\mathrm{U} / \mathrm{Th}$ timescale and its impact on understanding the Middle to Upper Paleolithic transition in Western Eurasia. Journal of Human Evolution 55, 772-781. 